\title{
Chinese or English? Writing Practices of Users from Mainland China on Instagram
}

\author{
Li Danling
}

\begin{abstract}
Studies on language use of non-native English speakers (NNES) are gaining momentum within the realm of computer-mediated communication (CMC). The present study, hence, addresses the NNES community in Mainland China through exploring their multilingual writing practices on the photo/video sharing mobile application, Instagram. Based on an approach of understanding literacies as a social practice, qualitative data are collected through exploratory observation of linguistic resources in the sites of 100 Instagramers and online interviews with 20 Mainland Chinese users. Findings demonstrate how Mainland Chinese users negotiate their language choices (Chinese/English) on Instagram and also indicate that linguistic practices are situated in a sociocultural context and could be influenced by a number of factors including users' situated linguistic ecology, audience awareness and the content of the post.
\end{abstract}

Index Terms - Instagram, language choice, code-switching, multilingual literacy practices.

\section{INTRODUCTION}

Many scholars express their concerns over the issues of English dominance and English as lingua franca on the Internet, regarding it as a threat to the status of other languages [1]. Meanwhile, statistics show that approximately two-thirds of Internet users are non-native English speakers (NNES) by 2016 [2] and millions of NNES users are participating in online activities today using their native languages or 'a mixture of languages'. There are also voices of localizing the Internet and imposing multilingualism on the web [3]. Under such a complex context, studies on the use of other languages, in particular, the NNES's native languages are gaining increasing popularity in the field of language use in computer-mediated communication (CMC) [4].

The Internet in Mainland China today is bombarded with various languages (e.g. English, simplified Chinese and varieties of Chinese) [5], which renders the online writing practices great value and authenticity to be explored. Hence, this study looks into Mainland Chinese users' writing practices on line, in particular, their language choices.

\section{A. The Context of the Study}

The present study investigates the multilingual writing practices of Mainland Chinese users on a mobile application (iOS and Android) - Instagram. Instagram is a popular photo/video capturing and sharing mobile application, with more than 500 million registered users by June, 2016 [2], also

Manuscript received January 6, 2017; revised June 17, 2017.

Li Danling is with the English Department, 3/F Fung King Hey Bldg, Chinese University of Hong Kong, Hong Kong (e-mail: lidanling@link.cuhk.edu.hk). providing a Web site where users can view posts on the Web interface.

Its popularity also leads to some academic studies shedding light on the photo content and its impact on different aspects including photography and photojournalism [6], teaching and learning [7] and etc. Nevertheless, relatively less studies pay attention to users' linguistic practices on Instagram, except the study of [8], in which Cantonese hashtags are examined and regarded as a form of representation of Hong Kong identities. This study aims to explore how Mainland Chinese users negotiate their language choices (English and Chinese) and switch codes in the online writing spaces by probing into their writing and linguistic practices on Instagram (including profile, hashtag, caption and comments) presented in Fig. 1.

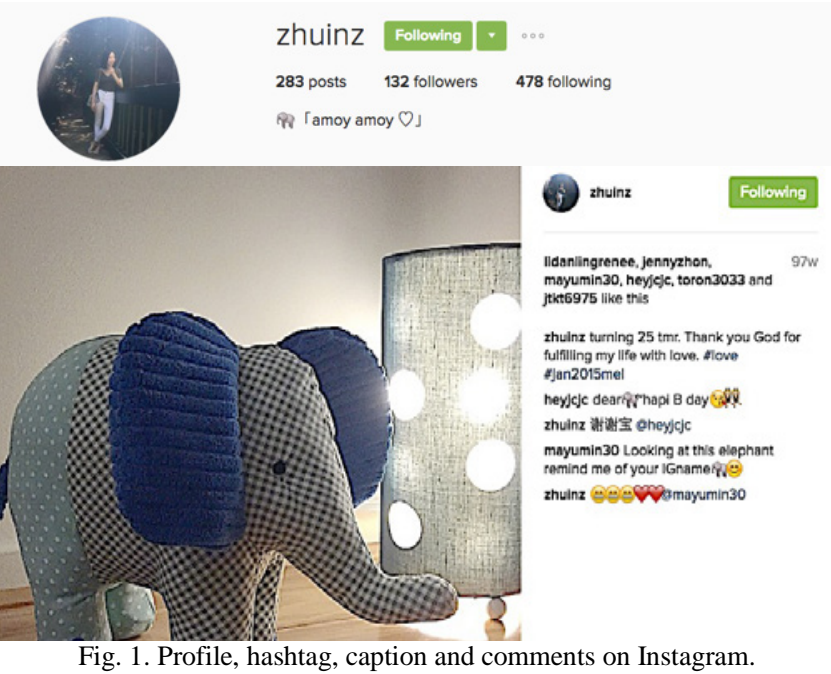

\section{B. Multilingual Writing Practices Online}

Instagram, with its large community, could be regarded as an emerging culture where people with different backgrounds are actively communicating and interacting with each other. Hence, this global online platform provides rich and thick data for language and literacy studies. Through the theoretical lens of 'literacy as a social practice' [9], the computer-mediated discourse (CMD), namely the writing and linguistic data on Instagram could be viewed as constructed and shaped in that sociocultural context where it is embedded.

Within the existing literature on CMC, the notion of 'language/code choice' and 'code-switching' (CS) are differently defined. Language choice is generally a broad conception concerning codes or linguistic recourses available to online participants. CS is defined as "dialogically interrelated by responding to previous, and contextualizing subsequent, contributions" [10]. Therefore, CS is more 
closely related with the online writing interactions (mainly Comments) [8].

Studies on online CS are well documented in [10] and [8], including different chosen platforms, languages, participants, methods and themes. Moreover, the structural patterns (insertional, alternational, intraword CS) and motivations of CS have also been comprehensively and succinctly summarized in [10] and [8]. Studies conducted in different sociocultural backgrounds reveal different reasons for CS, such as language preference [11], cultural value, ethnic identities [12], [13], [14], site's affordances [15], economic concerns [3] and etc. In a broader sense, factors accounting for users' different languages choices online were concluded in [8] as "situated language ecology of individual users, imagined audience, content of post and technological possibilities and constraints”.

In the light of the above studies, studies on language choice and CS need to be explored on a context-specific basis [16]. As not much has been known about Mainland Chinese users' writing practices online, the present study probes into the linguistic practices of Mainland Chinese users on Instagram and explores how they negotiate their language choices and switch codes online.

\section{Methodology}

A discourse-centered online ethnography [17] is adopted in this study, consisting of two phases of research: systematic observation of CMD and contacting the participants. I have been using Instagram for almost 3 years, regularly uploading pictures with captions and hashtags and also interact with other users using English and simplified Chinese on Instagram. My experience as an active user and follower offers me an insider's knowledge and a role as a participant observer in this study to gain a better understanding of participants' writing practices on Instagram [18]. Qualitative approach is adopted in this study to make an in-depth exploration in order to better address the research questions as follows:

1) How do Mainland Chinese users deploy their linguistic practices for meaning making on Instagram?

2) What are the factors affecting their language/code choice on Instagram?

\section{Data Collection Process}

Data collection spanned three months with daily checking and visiting Instagram. The main tools for data collection are exploratory observation [4] and email interview [19]. Collected data consists of screen-based data (including profile, hashtag, caption and comments) and user-based data (mainly through instant messages on Instagram and email-interview) [10]. Most participants prefer to communicate in Chinese. Hence, member checking has been conducted after the translation to ensure the validity of the results.

In the first phase, exploratory observation of different Instagram sites was conducted to the first 100 users that I came across and their latest 20 posts were examined. The selection of the 100 users followed two criteria [20]. First, participants should be users from Mainland China (explicitly stated in the profile). In order to gain deeper insights into participants' writing practices on Instagram, users from Hong Kong and Taiwan were not included because written Cantonese and traditional Chinese were not used in daily writing and communication in Mainland China. Second, they should be active users on Instagram (users' last post should be within one week from the date I started my exploratory observation).

In the second stage, exploratory observation and the subsequent email interview were both carried out to gain insights into users' language choices in writing practices on Instagram. In this stage, I identified 20 active multilingual users out of the 100 selected users and then examined each one of them by analyzing at least 100 posts with all the accompanied writing. Follow-up email interviews [14] were conducted respectively with the 20 chosen users to understand the complicated issues underpinning their writing practices (language choice/ CS). The selection criteria of the 20 users were: first, they should have at least 50 followers; second, they should contribute a lot in writing with at least 100 posts on Instagram (including profile, hashtag, caption and comments), as the posts were the primary data to be analyzed.

\section{MAINLAND ChINESE USERS' Writing PRACTICES ON INSTAGRAM}

A recursive process of the analysis of screen-based and user-based data was conducted to present users' multilingual practices on Instagram in four sections: profile, caption, hashtag and comments.

\section{A. Profile}

Profile in Instagram mainly consists of nickname and introduction. All screen names are required to be made of letters of the English alphabet, numbers, and symbols. Most observed Instagramers use their English names or names from Chinese Pinyin (or initial words of Chinese Pinyin) or the combination of the former two as the basis and add some symbols or characters to form their screen names, like fuxing810, versperyourname. Most of the introductions are written in English, indicating the location or presenting some self-descriptions (see Fig. 2).

\section{vesperyourname Following 00 fuxing810 Following $\ldots$ \\ Fig. 2. User's profile on Instagram.}

\section{B. Caption}

Caption is written and added to pictures or videos by the posters to explain the content or to express their feelings, which could be the main source for other users to comment. Many users write bilingual captions in English and Chinese. Some literally translate the Chinese into English sentence by sentence. Fig. 3 is a case of bilingual captions provided by vesperyourname, a Mainland Chinese student in Australia.

Vesperyourname first wrote in Chinese in the caption and translated it into English (Fig. 3). In her Chinese version, she put "ppt" and "presentation" embedded in the Chinese grammatical frame. 


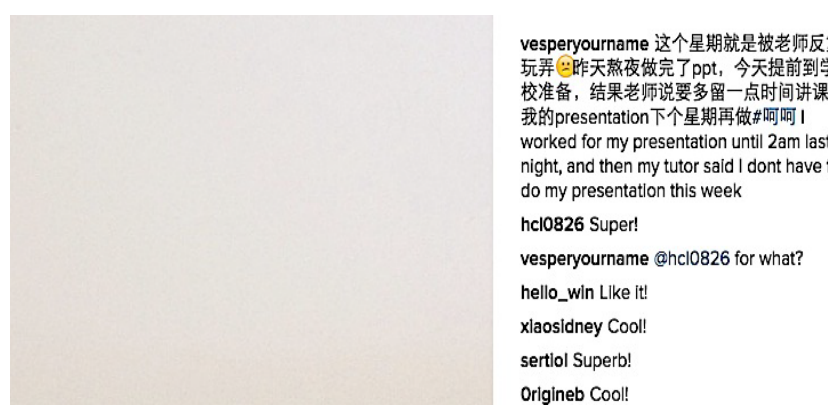

Fig. 3. Vesperyourname’s post

\section{Hashtag}

Hashtag is hyperlinked on Instagram, giving rise to "ambient affiliation" and facilitates "searchable talk" [8]. All the hashtags are analyzed taking considerations of the content of the posts [21]. Approximately two thirds of hashtags are written in English (some with their Chinese equivalents). Distinguished features emerge from the language use in hashtags. Users tend to extract words from the captions as hashtags and some will write bilingual hashtags both in English and Chinese indicating the same thing. Some Instagramers use specific kinds of words (e.g. location, mood) as hashtags. For instance, in Fig. 4, ray_yuan depicted the rainroom both in English and Chinese to tag the post. Also, he added \#inspiration, \#YUZMuseum and \#Shanghai to express his feelings and specify the location.

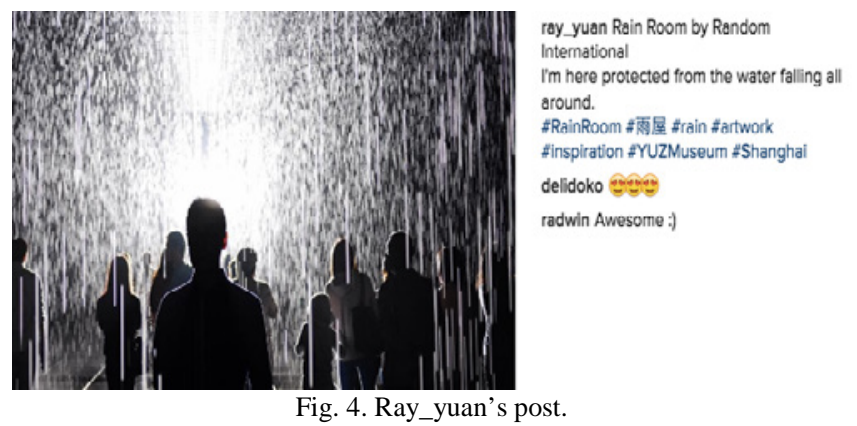

Another noteworthy linguistic use in hashtags is the intraword switching. Like Sara, a Mainland Chinese student in Italy, posted a world map on Instagram with a hashtag. In the hasgtag, she added the English inflectional suffix '-ing' to a Chinese verb phrase in order to present the present progressive tense.

\section{Comments}

Comments on Instagram are an important section for users to interact and socialize. The Mainland Chinese Instagramers in my study set their account open to all the users, welcoming comments in different languages. Therefore, most examples of inter- or intra-sentential CS [11] are elicited from this section after recursively examining the details of their multilingual use online. Users in my study intend to switch their language according to whom they are writing to. Below is an example (Fig. 5) posted by wangxiaolv, a photographer in Beijing.

Wangxiaolv replied sheirldawg in English knowing that she was a native English speaker whereas she responded puremolly in Chinese, because puremolly was her Chinese friend. As presented in Fig. 5, Puremolly used Chinese as the base language with the English word "Taco" inserted. In the last comment, zuoruo, her Chinese contact, started by using an English word "Taco", and switched to Chinese in the rest of the comment. This kind of CS complies with the System Morpheme Principle under the Matrix Language Frame (MLF) Model [22]. MLF Model suggests that lexical rather than grammatical/functional morphemes can be inserted as embedded language elements. In the above comments between wangxiaolv and her Mainland Chinese friends (Fig. 5), they used Chinese (L1) as the matrix language in the form of sentence structure, and inserted English (L2) as the embedded language in the form of lexical morphemes. The inserted morphemes are all lexical instead of grammatical ones.
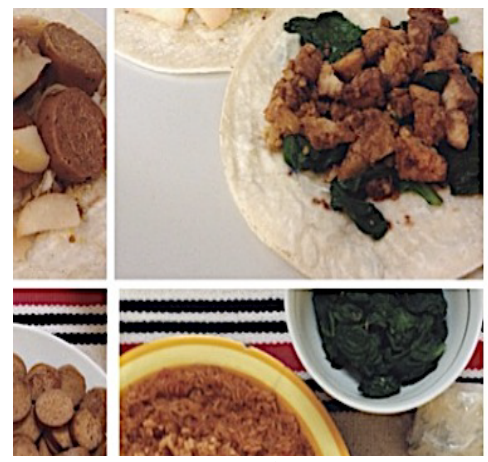

wangxiaolv \#WorldCup2014 themed Taco Donnerstag! Germany vs Argentina. German sausage with Sauerkraut and apples against Argentinian Insplred spinach, tempeh $w$ blue cheese. Guess who will win! \#Germany \#tacos shirldawg Every plateful looks dellcious. puremolly 感觉顿顿都在吃卷饼 wangxiaolv @shirldawg yes. Was really good. Sam made some guacamole. wangxiaolv @puremolly 因为每周四都是 taco之夜只有周四

zuoruo taco之夜! 祝早日拥有麻辣资之夜 肉夹馍之夜脏串儿之夜!

Fig. 5. Comments on Instagram.

\section{FACTORS INFLUENCING LANGUAGE CHOICE ON INSTAGRAM}

Based on the data presented above through exploratory observation and online interviews, Instagram could be viewed as a multilingual writing space for Mainland Chinese users to interact and socialize. Users' language/code choice on Instagram is influenced by various sociocultural factors. Based on the factors concluded in the study of [8], the present study address the second research question from the following three aspects.

\section{A. Situated Language Ecology}

Situated language ecology [8] refers to one's sociolinguistic and cultural backgrounds and their attitudes towards linguistic resources. In this study, all the users coming from Mainland China share the same writing system despite that they speak different varieties of Chinese. Most of the interviewed users acknowledge that English is becoming prevalent on the Internet and English is their first choice in writing profiles, captions and hashtags on Instagram. However, a user shows totally different attitudes towards the use of English on Instagram, stating that:

I am a patriot or you can say a nationalist. I will only say Chinese on Ins, you know.... I will try my best not to use English, but only if the one I am speaking to is a foreigner, you know. I will put tags, of course in Chinese to disseminate the political information.

His case is worth noting and what he said indeed matches what I observed. Chinese is his priority in writing things on Instagram and he only switched to English in the comments when he replied to a monolingual native speaker. 


\section{B. Audience Awareness}

Users' target audience [8] (their friends or the unknown public users) also influences their language choice. Users tend to respond to their audience using the same language they use to comment. Wangxiaolv explained, "The choice of language on Ins depends on which audience I want to speak to. Ins is more for my American friends and family as my Chinese friends are not so active on Ins.” Exceptions occur when users know that the audience could speak their native language (Chinese) and they probably switch to Chinese. Normally users are aware of the linguistic background of the audience they are responding to as they could indicate what language they are using by looking into their profiles, captions and etc.

Zoey and danraiy have different purposes of using bilingual posts, however, their common goal is to reach a wider and broader readership [21]. Danraiy mentioned that the reason why he used both English and Chinese hashtags was "to improve popularity of my post and gain more online media attention, so that I can have a broader social circle and get to know more new friends". In addition, Zoey explained, "audience is my main concern, for whom I use bilingual hashtags in different styles (catchwords, jargons, dialects, etc.) and top search term will be included." She also emphasized, "Instagram is a writing space for topic diffusion and spreading, in particular, for commercial purposes to attract your potential customers both locally and internationally."

\section{Content of Post}

Instagram is a photo (video) sharing application and photo (video) content exerts great influence on users' writing practices, especially the content closely related to cultural characteristics. For example, most of wangxialv's posts are described in English or at least with English equivalents. In describing one picture, however, she only used Chinese and Chinese Pinyin to depict and tag the post. In that picture, she's wearing blue, which was typical in Mao era and holding a book Chairman Mao Thought (translated from Chinese). She explained in the online interview that Chinese culture should be explained using Chinese to represent the cultural background.

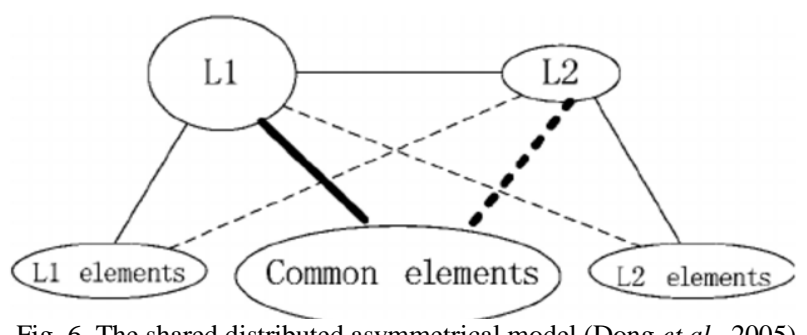

Fig. 6. The shared distributed asymmetrical model (Dong et al., 2005).

Chinese cuisine is another example for culture-related posts. In addition to the identity representation, some users explained that some of the Chinese cuisines were hard to translate. The Shared Distributed Asymmetrical Model [23] (Fig. 6) in Bilingualism theories can account for this phenomenon as both L1 and L2 have some specific elements, which can hardly find equivalents in the other language.

\section{CONCLUSION}

The present study demonstrates how Mainland Chinese users deploy linguistic sources in meaning making on Instagram by shedding light on their language choices. Adopting a social practice approach, this study elaborates on the online multilingual writing practices in different sections on Instagram (profile, caption, hashtag, comments). Situated linguistic ecology, audience awareness and the content of the post have emerged as the three major factors influencing users' language choices (English/Chinese) on Instagram after the recursive process of data analysis. Further study could be conducted under different contexts focusing on different linguistic communities.

\section{ACKNOWLEDGMENT}

I gratefully acknowledge the valuable suggestions provided by my supervisor Prof. Carmen Lee and many thanks to the research participants for their generous sharing and cooperation.

\section{REFERENCES}

[1] R. Phillipson and T. Skutnabb-Kangas, "Linguistic imperialism,” in Concise Encyclopedia of Sociolinguistics, New York: Elsevier, 2001, pp. 570-574.

[2] Internet World Stats. Internet Users by Country. (October 18, 2016). [Online]. Available: http://www.internetlivestats.com/internet-users-by-country/

[3] D. Dor, "From Englishization to imposed multilingualism: Globalization, the Internet, and the political economy of the linguistic code,” Public Culture, vol. 16, no. 1, pp. 97-118, Winter 2004.

[4] B. Danet and S. C. Herring, The Multilingual Internet: Language, Culture, and Communication Online, Oxford University Press, 2007.

[5] W. Zhang, "Multilingual creativity on China's Internet," World Englishes, vol. 34, no. 2, pp. 231-246, May 2015.

[6] M. Alper, "War on Instagram: Framing conflict photojournalism with mobile photography apps,” New Media \& Society, vol. 16, no. 8, pp. 1233-1248, September 2013.

[7] D. Salomon, "Moving on from Facebook using Instagram to connect with undergraduates and engage in teaching and learning," College \& Research Libraries News, vol. 74, no. 8, pp. 408-412, September 2013.

[8] K. C. Lee, Multilingualism Online, London and New York: Routledge. 2016.

[9] D. Barton and M. Hamilton, "Literacy practices” Situated Literacies, London: Routledge, 2000, ch.1, pp. 7-14.

[10] J. Androutsopoulos, "Code switching in computer-mediated communication,” Pragmatics of Computer-Mediated Communication, Berlin: Walter de Gruyter, 2013, ch. 27, pp. 667-694.

[11] J. Androutsopoulos, "Multilingualism, diaspora, and the Internet: Codes and identities on German-based diaspora websites," Journal of Sociolinguistics, vol. 10, pp. 520-547, August 2006.

[12] M. Durham, "Language choice on a Swiss mailing list," The Multilingual Internet: Language, Culture, and Communication Online, Oxford, U.K.: Oxford University Press. 2007, ch. 14. pp. 319-339.

[13] M. G. Kim, "Practicing multilingual identities: Online interactions in a Korean dramas forum,” International Multilingual Research Journal, vol. 10, no. 4, pp. 254-272, May 2016.

[14] K. C. Lee and D. Barton, "Constructing glocal identities through multilingual writing practices on Flickr. com ${ }^{\circledR}$, " International Multilingual Research Journal, vol. 5, no. 1, pp. 39-59, Feb. 2011.

[15] P. Seargeant, C. Tagg, and W. Ngampramuan, "Language choice and addressivity strategies in Thai-English social network interactions," Journal of Sociolinguistics, vol. 16, no. 4, pp. 510-531, September 2012.

[16] J. Blommaert, The Sociolinguistics of Globalization, Cambridge, UK: Cambridge University Press, 2001.

[17] J. Androutsopoulos. Potentials and limitations of discourse-centred online ethnography. [Online]. Available: http://www.languageatinternet.org/articles/2008/1610

[18] D. Barton and K. C. Lee, Language Online: Investigating Digital Texts and Practices, Routledge, 2013.

[19] C. Hine, Ethnography for the Internet: Embedded, Embodied and Everyday, Bloomsbury Publishing, 2015. 
[20] L. F. Perry, Research in Applied Linguistics: Becoming a Discerning Consumer, New York: Routledge, 2011.

[21] D. Barton, "Tagging on Flickr as a social practice," Discourse and Digital Practices, Doing Discourse Analysis in the Digital Age, New York: Taylor \& Francis, 2015, ch. 4, pp. 48-80.

[22] C. Myers-Scotton, Duelling Languages: Grammatical Structure in Code Switching, Oxford: Clarendon Press, 1993, ch. 4, p. 83.

[23] Y. Dong, S. Gui, and B. MacWhinney, "Shared and separate meanings in the bilingual mental lexicon," Bilingualism: Language and Cognition, vol. 8, no. 3, pp. 221-238, December 2005.

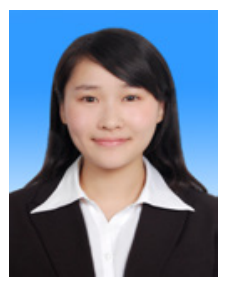

Li Danling was born in Mainland China, 1990. She graduated from Shanghai International Studies University (MA degree) in English linguistics and literature in Shanghai, China. Currently, she is an M. Phil student in Applied English Linguistics in the English Department at the Chinese University of Hong Kong.

She has worked in Fuzhou No. 4 Middle school for a year and a half since 2012, as an English teacher. She published An investigation on the professional stress of newly appointed teachers in primary school on the Journal of Changchun Education Institute in 2014 and A research on the English teaching modes of high school and social training institute on Journal of Education for Chinese After-school in 2011. Her research interests include literacy practices, English academic writing and qualitative method. 